psychopraxis. neuropraxis $2019 \cdot 22: 108-113$ https://doi.org/10.1007/s00739-019-0560-0 Online publiziert: 2. April 2019

(c) Der/die Autor(en) 2019
Stephan Listabarth · Daniel König · Andrea Gmeiner · Andreas Wippel · Benjamin Vyssoki

Klinische Abteilung für Sozialpsychiatrie, Universitätsklinik für Psychiatrie und Psychotherapie, Medizinische Universität Wien, Wien, Österreich

\section{Psychiatrische Behandlung eines alkoholabhängigen Patienten mit Lebererkrankung im Endstadium}

\author{
Kasuistische Darstellung
}

\section{Einleitung}

Alkoholabhängigkeit sowie die damit assoziierten Folgeerkrankungen sind von beträchtlicher gesundheits- und gesellschaftspolitischer Bedeutung. Vermehrter Alkoholkonsum kann eine Reihe von Erkrankungen bedingen, wobei fortgeschrittene Lebererkrankungen (z. B.: Leberzirrhose) hier von besonderer Bedeutung sind. So waren im Jahr 2010 1,8\% aller Todesfälle in Zentraleuropa auf alkoholbedingte Leberzirrhosen (ALZ) zurückzuführen [1]. Weltweit ist Alkoholkonsum für jährlich 3,3 Mio. Todesfälle $(5,9 \%$ aller Todesfälle) verantwortlich und gemessen an verlorenen Lebensjahren („disability-adjusted life years“ [DALYs]) beträgt der Anteil an alkoholassoziierten Erkrankungen 5,1\% an der gesamten globalen Belastung durch Erkrankungen und Verletzungen (139 Mio. DALYs) [2]. Zusätzlich ist die Lebenserwartung bei Patienten mit ALZ mit einer kumulativen 10-Jahres-Mortalität von 58,1\% [3] deutlich verkürzt. Die einzige kurative Therapie für diese Erkrankung ist eine Lebertransplantation. Abgesehen von der Komplexität und den Risiken eines solchen Eingriffs, müssen alkoholabhängige Patienten bei Transplantationszentren eine abstinente Phase von mindestens 6 Monaten vorweisen können, um für eine Transplantation gelistet zu werden [4]. Diese Regelung stellt gemeinsam mit der meist niedrigen Therapieadhärenz in diesem Patientenkollektiv eine Herausforderung dar. Die klinischen Folgen einer ALZ reichen von milderen Sympto- men wie Spider naevi, Palmarerythem, Ikterus, Mundwinkelrhagaden und Lackzunge zu schwerwiegenderen Komplikationen wie Aszites, Störungen des Gerinnungssystems oder einer hepatischen Enzephalopathie bis hin zu potenziell tödlichen Ereignissen wie eine spontan bakterielle Peritonitis, einem malignen hepatozellulären Karzinom oder einer stark erhöhten Wahrscheinlichkeit eine Sepsis zu erleiden [5]. Zusätzlich zu diesen somatischen Problemstellungen ist auch die psychiatrische Behandlung der Alkoholabhängigkeit, vor allem in Bezug auf die Pharmakotherapie, in dieser Patientengruppe mit eingeschränkter Leberfunktion limitiert.

\section{Falldarstellung}

In dem nun dargestellten Fall handelt es sich um einen 59-jährigen Patienten, der ursprünglich am 24.10.2018 auf der Universitätsklinik für Chirurgie, wegen einer fortschreitenden Aszites aufgenommen wurde. Während des Aufenthalts auf der chirurgischen Abteilung wurde der Patient bereits aufgrund von Alkoholentzugssymptomen konsiliarpsychiatrisch betreut und auf eine initiale Entzugsmedikation von $75 \mathrm{mg}$ Oxazepam pro Tag eingestellt und erhielt eine Substitutionstherapie mittels $600 \mathrm{mg}$ Thiamin (Vitamin B1) i.v. über 3 Tagesdosen verteilt für insgesamt 5 Tage.

Während des Aufenthalts an der chirurgischen Abteilung kam es im Zuge eines ausgeprägten Alkoholentzugssyndroms zu einem Sturz, der aufgrund einer bestehenden Antikoagulation mit Clopidogrel, mittels kranialer Computertomographie abgeklärt wurde. Dabei zeigte sich kein Hinweis auf eine intrakranielle Blutung. Wegen einer radiomorphologisch suszipierten Pneumonie und einer Fieberzacke, die sich während des Aufenthalts auf der chirurgischen Station ereignete, wurde eine Antibiose mit Moxifloxacin etabliert.

An der Universitätsklinik für Chirurgie wurden neben einer weiterführenden Abklärung der Leber mittels Lebervenenkatheter und Biopsie auch eine Gastroskopie sowie Koloskopie durchgeführt, welche bis auf pflastersteinartige Aspekte und einzelne, blande Sigmadivertikel keine Auffälligkeiten ergaben. Das Ergebnis der Lebervenenkatheteruntersuchung zeigte das Bild einer subklinischen, portalen Hypertension bei einem „hepatic venous pressure gradient" (HPVG) von $6 \mathrm{~mm} \mathrm{Hg}$ (Normwert 2-5 mm Hg). Die Histologie zeigte eine abszendierende Cholangitis, eine Steatohepatitis bei periportaler und perizellulärer Fibrose, sowie eine geringe Siderose im Parenchym. In mäßigem Allgemeinzustand wurde der Patient schließlich am 02.11.18 auf die Universitätsklinik für Psychiatrie und Psychotherapie transferiert, um den Alkoholentzug fortzusetzen und mit einer weiterführenden psychiatrischen Therapie zu beginnen.

Anamnestisch gab der Patient an, seit dem 35. Lebensjahr Alkohol zu konsumieren. Seit dem 50. Lebensjahr habe sich der Alkoholkonsum deutlich gesteigert und betrug seitdem im Durchschnitt 0,51 
hochprozentige (40\%) alkoholische Getränke täglich - einem Konsum von etwa $160 \mathrm{~g}$ reinen Alkohols pro Tag entsprechend. Nach einer Omega-Loop-BypassOperation bei Adipositas per magna im Jahr 2013 war der Alkoholkonsum des Patienten kurzeitig etwas reduziert, schrittweise steigerte sich dieser aber wieder auf das präoperative Niveau. Im Januar 2018 wurde bei dem Patienten schließlich durch das Krankenhaus Rudolfstiftung eine Leberzirrhose erstdiagnostiziert.

Aufgrund dieser Diagnose reduzierte der Patient seinen Alkoholkonsum auf $100 \mathrm{ml}$ hochprozentige alkoholische Getränke pro Tag - einem täglichen Konsum von etwa $33 \mathrm{~g}$ reinen Alkohols entsprechend. Der Patient hatte bisher noch nie psychiatrische oder psychotherapeutische Betreuung in Anspruch genommen. Zusätzliche, bereits vorbekannte, körperliche Komorbiditäten waren eine arterielle Hypertonie, ein insulinabhängiger Diabetes mellitus (IDDM), der Verdacht auf einen leichten Schlaganfall im Jahr 2008 sowie der Zustand nach Koronar-Stent-Implantation bei koronarer Herzkrankheit (KHK) im Jahr 2017. Seit Januar 2018 war der Patient wiederholt wegen einer Aszites in Behandlung gewesen. Eine dieser Episoden führte letztlich zur Aufnahme auf der Universitätsklinik für Chirurgie.

Der medikamentös gestützte Alkoholentzug wurde bei diesem Patienten auch nach Übernahme auf die Universitätsklinik für Psychiatrie weiter mit Oxazepam durchgeführt. Dieser Wirkstoff weist einige Vorteile in der klinischen Anwendung gegenüber anderen Vertretern der Benzodiazepine auf. Diese sind speziell bei Patienten mit Lebererkrankungen relevant. So wird Oxazepam in der Leber lediglich glukuronidiert und nicht über das CYP-Enzym-System metabolisiert, welches bei fortgeschrittenen Lebererkrankungen meist wesentlich früher in ihrer Funktionalität reduziert ist als die Glukuronidierung [6, 7]. Alternativ hätte auch Lorazepam eingesetzt werden können, das ebenfalls lediglich glukoronidiert wird.
Somatische Aspekte der Behandlung

Im weiteren Verlauf war es wichtig, weitere durch die Leberfunktionsstörung bedingte internistische Komplikationen frühzeitig $\mathrm{zu}$ diagnostizieren und adäquat $\mathrm{zu}$ behandeln. Insbesondere sind auf eine weitere Verschlechterung der Lebersynthesefunktion, Anämie, Thrombozytopenie, Elektrolytentgleisungen, kompromittierte Gerinnung und Ammoniakerhöhung zu achten.

Als grundlegende Diagnostik wurden im gegenständlichen Fall regelmäßige Laborkontrollen durchgeführt. In diesen zeigte sich neben einer normochromen normozytären Anämie, eine Thrombozytopenie sowie erhöhte Leberenzymwerte (GOT, GPT, $\gamma$-GT), ein erhöhtes Bilirubin und eine verschlechterte Gerinnung im Sinne einer erhöhten INR (International Normalized Ratio) und Verlängerung des Normotests.

Die Anämie ist bei Patienten mit Alkoholabhängigkeit einerseits oft durch einen Mangel an Vitamin B12 und Folsäure sowie durch einen direkten myelodepressiven Effekt des Ethanols bedingt [8]. Dies kann nicht nur zu einer Anämie führen, sondern auch zu einer generellen Panzytopenie. Liegt dann auch noch eine fortgeschrittene Lebererkrankung vor, ist das Immunsystem des $\mathrm{Pa}$ tienten über multiple Mechanismen reduziert und die Infektanfälligkeit dieser Patienten ist massiv erhöht [5]. Der vorzustellende Patient hatte zum Zeitpunkt der Transferierung 7,70 G/l Leukozyten und ein Hämoglobin von 8,3g/dl. Da sich die Erythropoese im Laufe des Entzugs erfahrungsgemäßrelativ rasch erholt und normalisiert - auf eine Substitution bei zusätzlich vorhanden Eisenmangel ist zu achten - wurde diesbezüglich auf eine unmittelbare Therapie verzichtet. Im Zuge des Weiteren stationären Verlaufs verbesserten sich die entsprechenden Parameter und der Patient konnte schließlich mit einem Hämoglobin von 9,2 g/dl entlassen werden.

Die deutlich erhöhten Werte der Leberenzyme (GOT: 122 U/l, GPT: 46U/l) standen im Einklang zur psychiatrischen Grunderkrankung. Ebenso der De-RitisQuotient >2 (2,65). - Dieser spielt eine wichtige Rolle in der Differenzialdiagnose von Lebererkrankungen und wird aus dem Verhältnis zwischen GOT und GPT beziehungsweise AST und ALT berechnet. Liegt der De-Ritis-Quotient zwischen 1 und 2 ist das ein möglicher Hinweis auf vermehrten Alkoholkonsum, Werte über 2 sprechen für eine akute alkoholbedingte Hepatitis. Werte kleiner 1 wiederum sprechen für eine Lebererkrankung viraler Genese [9].

\section{》) Ein De-Ritis-Quotient \\ über 2 weist auf eine akute alkoholbedingte Hepatitis hin}

Die INR wird maßgeblich durch die in der Leber synthetisierten Gerinnungsfaktoren II, V, VIII und X bestimmt und stellt daher einen Verlaufsparameter für die Syntheseleistung der Leber dar. Diesen Zusammenhang kann man sogar diagnostisch nutzen, in dem eine parenterale Gabe von Vitamin Kverabreicht wird und dann mit einem ausreichenden Abstand von mindestens $24 \mathrm{~h}$ die Bestimmung der INR wiederholt wird. Normalisieren sich die Gerinnungstests nicht, so ist eine Leberzellinsuffizienz ursächlich für die gestörte Gerinnung und nicht eine Resorptionsstörung oder ein Vitamin-K-Mangel [10]. Beim vorzustellenden Fall kam es zu einer geringgradigen Verbesserung der Thromboplastinzeit bzw. des QuickWerts nach parenteraler Vitamin-K-Gabe. Es wurde daher von einer ausreichend vorhandenen Lebersyntheseleistung ausgegangen.

Ebenfalls im Zusammenhang mit der Syntheseleistung der Leber sind verminderte Albumin-Spiegel bzw. verringerte Gesamteiweißwerte im Serum zu sehen. Das klinische Korrelat zum Albumin-Mangel $(26,8 \mathrm{~g} / \mathrm{l})$ im vorzustellenden Fall waren beidseits mäßige Beinödeme sowie ein Perikarderguss. Daher erhielt der Patient in den ersten Tagen nach der Transferierung parenteral zusätzlich zu Vitamin K auch Humanalbumin. Die ebenfalls in Laborkontrollen festgestellten Elektrolytentgleisungen (Hypokäliämie, Hyponatriämie und Hypochloridämie) wurden durch entsprechende orale Substitution ausgeglichen. 
Ein weiterer wichtiger Laborparameter, der bestimmt wurde, bei diesem Fall allerdings im Referenzbereich lag, ist Ammoniak. Die Substanz ist ein Marker, der mit dem Auftreten einer hepatischen Enzephalopathie (HE) assoziiert sein kann. Diese Komplikation ist von psychiatrischer Relevanz, da sie klinisch, abhängig vom Schweregrade, primär durch Bewusstseinsstörungen von Orientierungsverlust und Verwirrtheit bis hin zum Koma gekennzeichnet ist. Auch wenn die Pathophysiologie der HE noch nicht eindeutig geklärt ist, spielt Ammoniak dabei definitiv eine wichtige Rolle. Erhöhte Serumspiegel wirken neurotoxisch und bewirken eine Inhibition der exzitatorischen Neurotransmission und können bei einem abrupten Anstieg, zum Beispiel im Rahmen einer akuten alkoholbedingten Hepatitis, auch ein Hirnödem auslösen [11]. Die klinische Einteilung erfolgt nach den WesthavenKriterien. Hierbei ist zu beachten, dass speziell bei Patienten mit Leberzirrhose die Symptome einer HE mild ausgeprägt und schwer von alkoholbedingten kognitiven Defiziten zu unterscheiden sein können. Dennoch kann diese Form einer HE verantwortlich für Stürze, Müdigkeit und inadäquates Verhalten der Patienten sein [11].

Anhand spezifischer Klassifikationen, wie dem Child-Pugh-Score oder dem Model-of-End-Stage-Liver-Disease(MELD)-Score, der ursprünglich zur Objektivierung einer Transplantationsindikation entwickelt wurde, kann das Stadium der Lebererkrankung evaluiert und eine Prognose angegeben werden. Der MELD-Score wird berechnet aus Serumkreatinin, Serumbilirubin und INR.

\section{》) Ammoniak ist bei einer hepatischen Enzephalopathie ein wichtiger Laborparameter}

Demgegenüber werden beim ChildPugh-Score zusätzliche klinische Parameter wie das Vorliegen einer Aszites oder einer hepatischen Enzephalopathie bewertet. Diese Klassifikationen zeigen auch eindrucksvoll die schlechte Prognose dieser Erkrankungen auf. So liegt

psychopraxis. neuropraxis 2019 -22:108-113 https://doi.org/10.1007/s00739-019-0560-0 c) Der/die Autor(en) 2019

\section{S. Listabarth · D. König · A. Gmeiner · A. Wippel · B. Vyssoki}

\section{Psychiatrische Behandlung eines alkoholabhängigen Patienten mit Lebererkrankung im Endstadium. Kasuistische Darstellung}

\section{Zusammenfassung}

Dieser Fallbericht gibt einen Überblick über die Aspekte des multidisziplinären Managements eines alkoholabhängigen Patienten mit Lebererkrankung im Endstadium. Wir berichten von einem 59jährigen Patienten, der für einen stationären, medikamentös-gestützten Alkoholentzug aufgenommen worden war. Der Patient litt an einer dekompensierten, alkoholinduzierten Leberzirrhose. Die ursprüngliche Aufnahme erfolgte an einer chirurgischen Abteilung aufgrund einer zunehmenden Aszites und einer generellen Verschlechterung des Allgemeinzustands. Nach konsiliarpsychiatrischer Erstvorstellung und Einleitung einer Behandlung bei Alkoholentzug wurde er rasch an die Station 04A der Universitätsklinik für Psychiatrie und Psychotherapie, Klinische Abteilung für Sozialpsychiatrie übernommen. Der klinische und wissenschaftliche Schwerpunkt der Station 04A ist die Behandlung alkoholabhängiger Patienten. Neben der internistischen Behandlung der Folgen der Leberfunktionsstörung wurde ein schrittweiser Alkoholentzug mithilfe von Benzodiazepinen erfolgreich durchgeführt sowie eine Anti-craving-Therapie mit Baclofen etabliert.

\section{Schlüsselwörter}

Suchterkrankung · Sozialpsychiatrie ·

Leberzirrhose · Entzug · Aszites

\section{Psychiatric Treatment of an Alcohol-Dependent Patient with End Stage Liver Disease. Case Report}

\section{Abstract}

This case report delivers a concise overview of the multidisciplinary management of patients with alcohol use disorder and end-stage liver disease: We report the case of a 59-yearold patient, suffering from end stage liver disease as a result of alcohol use disorder, undergoing a pharmacological-supported alcohol withdrawal. The patient was initially admitted to the surgical department due to progressing ascites and a reduced general condition. However, as soon as the patient was stabilized and the alcohol withdrawal was initiated, he was transferred to ward 04A Department of Psychiatry and Psychotherapy,
Clinical Division of Social Psychiatry. The clinical and scientific focus of this ward is the management of patients with alcohol use disorder. Besides treating the internistic conditions associated with the end-stage liver disease of this patient, a step-wise alcohol withdrawal was conducted and an anti-craving therapy with baclofen was established.

\section{Keywords}

Addiction - Social psychiatry - Liver cirrhosis . Substance withdrawal · Ascites die 2-Jahres-Mortalität bei einem ChildPugh-Stadium C bei $75 \%$ und die 3-Monats-Mortalität bei einem MELD-Score von $30-39$ bei $52,6 \%$ und bei Werten über 40 sogar bei $71,3 \%$. Im gegenständlichen Fall hatte der Patient einen Child-Pugh-Score entsprechend einem Stadium B (40\% 2-Jahres-Mortalität) und einen MELD-Score von 20 Punkten (19,6\% 3-Monats-Mortalität).

\section{Diagnostische Maßnahme: Elastizitätsmessung}

Als weitere diagnostische Maßnahme zur direkten Beurteilung des Zustandes der Leber wurde im vorgestellten Fall eine sog. Elastizitätsmessung (Fibroscan ${ }^{\circledR}$ [Echosens, Paris, Frankreich]) durchgeführt. Bei dieser Untersuchung werden mit einem Schallkopf Vibrationsbewegungen erzeugt, die transdermal auf das Leberparenchym abgegeben werden. Eine Ultraschallsonde analysiert die reflektierenden Wellen und kann die Verformung, d.h. die Elastizität 


\begin{tabular}{|c|c|}
\hline $\begin{array}{l}\text { Keine Dosisanpassung } \\
\text { notwendig }\end{array}$ & (Levo)Milnacipran \\
\hline $\begin{array}{l}\text { Keine Dosisanpassung } \\
\text { bei geringer-mittlerer } \\
\text { Einschränkung }\end{array}$ & $\begin{array}{l}\text { Escitalopram } \\
\text { Vortioxetin }\end{array}$ \\
\hline Eingeschränkt nutzbar & $\begin{array}{l}\text { Bupropion } \\
\text { Citalopram } \\
\text { Venlafaxin } \\
\text { Fluoxetin } \\
\text { Mirtazapin } \\
\text { Paroxetin } \\
\text { Amitriptylin }\end{array}$ \\
\hline Vermeiden & Duloxetin \\
\hline Wenig Daten vorhanden & Sertralin \\
\hline
\end{tabular}

des Leberparenchyms quantifizieren. Je höher der gemessenen Werte (in Kilopascal $[\mathrm{kPa}])$, desto unelastischer ist das Organ. Im Fall der alkoholbedingten Leberzirrhose resultiert diese Steifigkeit aus einer massiven Einlagerung von Bindegewebsfasern in das Parenchym, der sog. Fibrosierung. Die Elastizitätsmessung ermöglicht es, spezifisch zwischen den einzelnen Stadien der Fibrosierung (F0-F4) zu differenzieren. Wichtig hierbei ist jedoch, dass eine verringerte Elastizität nicht exklusiv durch eine Zirrhose bedingt sein muss, sondern unter anderem auch durch systemische Erkrankungen mit Leberbeteiligung (Amyloidose) verursacht sein kann oder eine infektiöse Genese (Hepatitis A/B) haben kann. Daher sind die Ergebnisse dieser Untersuchung nur in Zusammenschau mit den assoziierten Labormarkern und der Klinik interpretierbar [12]. Beim vorgestellten Patienten konnte durch die Elastizitätsmessung der Leber die suspizierte Leberzirrhose im Endstadium F4 sowie eine ausgeprägte Steatosis hepatis bestätigt werden $(34,3 \mathrm{kPa}$, CAP: 327$)$.

\section{Psychiatrische Aspekte der Behandlung}

Eine medikamentöse Anti-craving- und Rückfallprophylaxe bei Patienten mit fortgeschrittener Lebererkrankung gestaltet sich schwierig, da bei den einzigen beiden in Österreich zugelassenen Medikamenten, Naltrexon und Acamprosat, keine ausreichende Sicherheit für den Einsatz bei schweren Leberschädigungen angegeben wird [13]. Erstaunlicherweise gibt es trotz der häufig zu erwartenden Assoziation von Alkoholabhängigkeit und fortgeschrittener Lebererkrankung nur wenig evidenzbasierte Forschung über eine suffiziente Anti-craving-Therapie für dieses Patientenkollektiv. Erstmalig konnten in einer randomisierten doppelblinden placebokontrollierten Studie Addolorato et al. [14] eine signifikante Steigerung der Abstinenzrate unter einer Therapie mit Baclofen nachweisen. Baclofen wird initial mit einer Dosis von 3 Einzeldosen von $5 \mathrm{mg}$ pro Tag etabliert und nach 3 Tagen auf eine Zieldosis von $30 \mathrm{mg}$ pro Tag auf 3 Einzeldosen gesteigert [15]. Allerdings ist die Evidenzlage zur Wirksamkeit von Balcofen limitiert und ein systematischer Cochrane-Review kommt sogar zu der Schlussfolgerung, dass Baclofen in Bezug auf Rückfallsrate und CravingSymptomatik gegenüber Placebo nicht überlegen ist [16]. Aufgrund der doch deutlich beeinträchtigten Leberfunktion des Patienten wurde noch während des stationären Aufenthalts des Patienten nach oben beschriebenen Schema die Therapie mit Baclofen als Anti-cravingund Rückfallprophylaxe begonnen. Bei guter klinischer Verträglichkeit wurde diese Dosierung auch als Entlassungsmedikation weiter verordnet.

Da der Patient im Verlauf des stationären Aufenthalts über Einschlafstörungen klagte, wurde die bereits bestehende Medikation mit $150 \mathrm{mg}$ Trazodon retard abends auf eine Dosis von $200 \mathrm{mg}$ gesteigert, worauf der Patient über eine suffizient schlaffördernde Wirkung berichtete. Prinzipiell ist beim Einsatz aller Antidepressiva bei Patienten mit ALZ Vorsicht geboten, da die eingeschränkte Metabolisierung der Leber zu einer veränderten Pharmakokinetik führen kann. Eine Sonderposition unter den Antidepressiva nimmt hier das Milnacipran ein, das in der Leber hauptsächlich glukuronidiert und überwiegend renal eliminiert wird. Bei allen anderen Vertretern der SSRIs („Selective Serotonin Reuptake Inhibitor") oder SNRIS („SerotoninNoradrenalin Reuptake Inhibitor") und der TZA (,trizyklische Antidepressiva“) ist ein Einsatz nur unter Dosisanpassung empfohlen, Duloxetin und Venlafaxin sind hingegen bei schwerer Leberschädigung kontraindiziert [17, 18]. Eine Übersicht über einige ausgewählte Psychopharmaka und ihre Anwendbarkeit bei fortgeschrittener Lebererkrankung gibt • Tab. 1.

\section{Zusammenfassung}

Nach einem erfolgreich durchgeführten medikamentösen Alkoholentzug, einer Stabilisierung des somatischen $\mathrm{Zu}$ standsbilds und der Etablierung einer Anti-craving- und Rückfallprophylaxe, konnte der Patient nach 25 Tagen stationären Aufenthalts in deutlich gebesserten Allgemeinzustand nach Hause entlassen werden. Eine engmaschige psychiatrische Betreuung für die Zeit nach dem Aufenthalt wurde organisiert. Ein telefonisches Follow-up-Gespräch fand etwa 3 Monate nach der Entlassung statt. Der Patient berichtete, in einem guten Gesundheitszustand und weiterhin abstinent $\mathrm{zu}$ sein.

\section{Diskussion}

Anhand dieses Fallberichts berichten wir über die vielfältigen Aspekte in der Behandlung von alkoholabhängigen Patienten mit fortgeschrittener Lebererkrankung. Begleitend durch die theoretischen Erläuterungen im Zusammenhang mit dem klinischen Verlauf in der speziellen Kasuistik werden die notwendigen diagnostischen und therapeutischen Maßnahmen beschrieben.

\section{》) Bei eingeschränkter Leber- funktion ist die Hepatotoxizität von Psychopharmaka zu beachten}

In Bezug auf die psychopharmakologische Therapie ist die eingeschränkte Leberfunktion und die damit einhergehende veränderte Metabolisierung beziehungsweise potenziell hepatotoxische Wirkung von diversen Psychopharmaka zu beachten. Die für diese Patienten infrage kommenden Medikamente im Bereich des medikamentös gestützten Alkoholentzugs, der Anti- 
craving- und Rückfallprophylaxe sowie der Behandlung von etwaigen psychiatrischen Komorbiditäten wurden in diesem Artikel dargestellt. Für die Prognose der Patienten ist neben einer engmaschigen internistischen Betreuung vor allem hinsichtlich der 6-Monats-Regel zur Listung in Transplantationszentren eine weiterführende und umfassende psychiatrische und psychotherapeutische Betreuung und das Aufbauen eines supportiven psychosozialen Netzwerks von entscheidender Bedeutung.

\section{Fazit für die Praxis}

- Generell ist bei der psychopharmakologischen Pharmakotherapie bei Patienten mit fortgeschrittener Lebererkrankung die Metabolisierung und Hepatotoxizität zu beachten.

- Der medikamentös gestützte Alkoholentzug sollte mit Oxazepam oder Lorazepam durchgeführt werden.

- Als Anti-craving- und Rückfallprophylaxe bei fortgeschrittener Lebererkrankung kann Baclofen eingesetzt werden.

- Potenziell lebensgefährliche Komplikationen sind Varizenblutungen und fulminant verlaufende Infektionen.

- Engmaschige klinische Kontrollen sowie eine multidisziplinäre Betreuung dieser Patienten sind notwendig.

- Im fortgeschrittenen, irreversiblen Stadium einer Lebererkrankung ist eine Transplantation die letzte kurative Therapieoption.

- Eine absolute Alkoholabstinenz ist hinsichtlich der Prognose der entscheidende Faktor.

\section{Korrespondenzadresse}

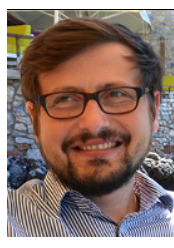

(c) Privat

Assoc. Prof. PD Dr.Dr.

Benjamin Vyssoki

Klinische Abteilung

für Sozialpsychiatrie,

Universitätsklinik

für Psychiatrie und

Psychotherapie, Medizinische

Universität Wien

Währinger Gürtel 18-20,

1090 Wien, Österreich

benjamin.vyssoki@

meduniwien.ac.at

Funding. Open access funding provided by Medical University of Vienna.

\section{Einhaltung ethischer Richtlinien}

Interessenkonflikt. S. Listabarth, D. König, A. Gmeiner und A. Wippel geben an, dass kein Interessenkonflikt besteht. B. Vyssoki hat Vortragshonorare der Firma Germania erhalten.

Für diesen Beitrag wurden von den Autoren keine Studien an Menschen oder Tieren durchgeführt. Für die aufgeführten Studien gelten die jeweils dort angegebenen ethischen Richtlinien. Für Bildmaterial oder anderweitige Angaben innerhalb des Manuskripts, über die Patienten zu identifizieren sind, liegt von innen und/oder ihren gesetzlichen Vertretern eine schriftliche Einwilligung vor.

Open Access Dieser Artikel wird unter der Creative Commons Namensnennung 4.0 International Lizenz (http://creativecommons.org/licenses/by/4.0/deed. de) veröffentlicht, welche die Nutzung, Vervielfältigung, Bearbeitung, Verbreitung und Wiedergabe in jeglichem Medium und Format erlaubt, sofern Sie den/die ursprünglichen Autor(en) und die Quelle ordnungsgemäßnennen, einen Linkzur Creative Commons Lizenz beifügen und angeben, ob Änderungen vorgenommen wurden.

\section{Literatur}

1. Rehm J, Samokhvalov AV, Shield KD (2013) Global burden of alcoholic liver diseases. J Hepatol 59(1):160-168

2. WHO (2019) Management of substance abuse-Alcohol. WHO Fact \& Figures. https://www. who.int/substance_abuse/facts/alcohol/en/. Zugegriffen: 15. Febr. 2019

3. Marot A, Henrion J, Knebel J-F, Moreno C, Deltenre P (2017) Alcoholic liver disease confers a worse prognosis than HCV infection and non-alcoholic fatty liver disease among patients with cirrhosis: an observational study. PLoS ONE 12(10):e186715

4. Marroni CA, Fleck AM, Fernandes SA, Galant LH, Mucenic M, de Mattos Meine MH, Mariante-Neto G, de Brandão ABM (2018) Liver transplantation and alcoholic liver disease: history, controversies, and considerations. World J Gastroentero 24(26):2785-2805

5. Gustot T, Fernandez J, Szabo G, Albillos $A$, Louvet A, Jalan R, Moreau R, Moreno C (2017) Sepsis in alcohol-related liver disease. J Hepatol 67(5):1031-1050

6. Perry EC (2014) Inpatient management of acute alcohol withdrawal syndrome. CNS Drugs 28(5):401-410

7. Leggio L, Lee MR (2017) Treatment of alcohol use disorder in patients with alcoholic liver disease. Am JMed 130(2):124-134

8. Smith C, Gasparetto M, Jordan C, Pollyea DA, Vasiliou V (2015) The effects of alcohol and aldehyde dehydrogenases on disorders of hematopoiesis. AdvExp Med Biol 815:349-359

9. Botros M, Sikaris KA (2013) The de ritis ratio: the tes of time. Clin Biochem Rev 34(3):117-130

10. Gressner A, Gressner O (2018) Koller-Test. In:Gressner A, Arndt T (Hrsg) Lexikon der Medizinischen Laboratoriumsdiagnostik. Springer Reference Medizin. Springer, Berlin, Heidelberg
11. Wijdicks EFM (2016) Hepatic encephalopathy. NEngl J Med 375(17):1660-1670

12. Sharma S, KhaliliK, Nguyen GC (2014) Non-invasive diagnosis of advanced fibrosis and cirrhosis. World J Gastroenterol 20(45):16820-16830

13. Stoklosa TM, Morley KC, Volovets A, Haber PS (2018) Pharmacotherapy for alcohol use disorde in the context of liver disease. Curr Addict Rep 5(2):287-296

14. Addolorato G, Leggio L, Ferrulli A, Cardone S, Vonghia L, Mirijello A, Abenavoli L, D’Angelo C, Caputo F, Zambon A, Haber PS, Gasbarrini G (2007) Effectiveness and safety of baclofen for maintenance of alcohol abstinence in alcohol-dependent patients with liver cirrhosis: randomised, double-blind controlled study. Lancet 370(9603):1915-1922

15. Addolorato G, Caputo F, Capristo E, Domenicali M, Bernardi M, Janiri L, Agabio R, Colombo G, Gessa GL, Gasbarrini $G$ (2002) Baclofen efficacy in reducing alcohol craving and intake: a preliminary doubleblind randomized controlled study. Alcohol Alcohol 37(5):504-508

16. Minozzi S, Saulle R, Rösner S (2018) Baclofen for alcohol use disorder. Cochrane Database Syst Rev 11:CD12557

17. Mullish BH, Kabir MS, Thursz MR, Dhar A (2014) Review article: depression and the use of antidepressants in patients with chronic liver disease or liver transplantation. Aliment Pharmacol Ther 40(8):880-892

18. Benkert O, Hippius H (2016) Kompendium de psychiatrischen Pharmakotherapie. Springer, Berlin, Heidelberg

Hinweis des Verlags. Der Verlag bleibt in Hinblick auf geografische Zuordnungen und Gebietsbezeichnungen in veröffentlichten Karten und Institutsadressen neutral. 
Hier steht eine Anzeige.

\section{曾 Springer}

